\title{
Commentary: Genome Sequence of Vibrio parahaemolyticus VP152 Strain Isolated From Penaeus indicus in Malaysia
}

\author{
Theodore Allnutt ${ }^{1,2}$, Chrystine Zou Yi Yan ${ }^{3,4}$, Tamsyn M. Crowley ${ }^{1,2,5}$ and Han Ming Gan ${ }^{3,4,6 *}$ \\ ${ }^{1}$ Bioinformatics Core Research Group, Deakin University, Geelong, VIC, Australia, ${ }^{2}$ School of Medicine, Centre for Molecular \\ and Medical Research, Deakin University, Geelong, VIC, Australia, ${ }^{3}$ School of Science, Monash University Malaysia, Petaling \\ Jaya, Malaysia, ${ }^{4}$ Genomics Facility, Tropical and Biology Multidisciplinary Platform, Monash University Malaysia, Petaling \\ Jaya, Malaysia, ${ }^{5}$ Poultry Hub Australia, University of New England, Armidale, NSW, Australia, ${ }^{6}$ Centre for Integrative, School \\ of Life and Environmental Sciences, Deakin University, Geelong, VIC, Australia
}

Keywords: Vibrio parahaemolyticus, taxonomy, genomics, shrimp, South East Asia

\section{A commentary on}

Genome Sequence of Vibrio parahaemolyticus VP152 Strain Isolated from Penaeus indicus in Malaysia

by Letchumanan, V., Ser, H.-L., Tan, W.-S., Ab Mutalib, N.-S., Goh, B.-H., Chan, K.-G., et al. (2016). Front. Microbiol. 7:1410. doi: 10.3389/fmicb.2016.01410

Vibrio parahaemolyticus is a marine gram negative bacterium that has been gaining significant attention in the shrimp aquaculture industry given its direct association with early mortality syndrome (EMS) or acute hepatopancreatic necrosis disease (AHPND) in shrimps (Soto-Rodriguez et al., 2015). Despite its significant threat to the industry, the genomic representation of shrimpassociated $V$. parahaemolyticus isolated from Malaysia or South East Asia in general is relatively low (Kondo et al., 2014; Yang et al., 2014; Foo et al., 2017). Letchumanan and colleagues recently reported the draft genome of $V$. parahaemolyticus VP152 isolated from a banana prawn in Malaysia (Letchumanan et al., 2016b). Strain VP152 was sequenced on the Illumina MiSeq and its whole genome sequence was deposited in DDBJ/EMBL/GenBank under the accession number and Bioproject ID of LCUL01000000 and PRJNA281142, respectively.

The G+C content for strain VP152 was reported to be $53.4 \%$ which is substantially higher than the average $\mathrm{G}+\mathrm{C}$ content of $V$. parahaemolyticus ( $45 \%$ ) (Kondo et al., 2014; Yang et al., 2014; Foo et al., 2017). A similarity search of house-keeping genes coded in the genome of strain VP152 showed best hits to members of the genus Citrobacter (data not shown). A subsequent phylogenomic analysis using PhyloPhlAN (Segata et al., 2013) clustered strain VP152 with members of the genus Citrobacter with strong SH-like local branch support (Figure 1A). In addition, similar to several Citrobacter strains, when searched against the complete genome of $V$. parahaemolyticus ATCC $17802^{\mathrm{T}}$, strain VP152 exhibited only modest genomic region with significant nucleotide homology to the $V$. parahaemolyticus reference genome (Figure 1B) (Alikhan et al., 2011). It is also worth noting that $V$. parahaemolyticus strain VP103 deposited in $\mathrm{DDBJ} / \mathrm{EMBL} / \mathrm{GenBank}$ under the accession number LBDB01000000 reported by the same group in a different data report (Letchumanan et al., 2016a) also showed the same phylogenomic affiliation to the genus Citrobacter instead of Vibrio.

Furthermore, a search in the NCBI bioproject database revealed that C. amalonaticus YG6 and C. amalonaticus YG8 with the Bioproject IDs of PRJNA292629 and PRJNA292637, respectively, were also sequenced by the same institute. This observation in addition to the monophyletic 

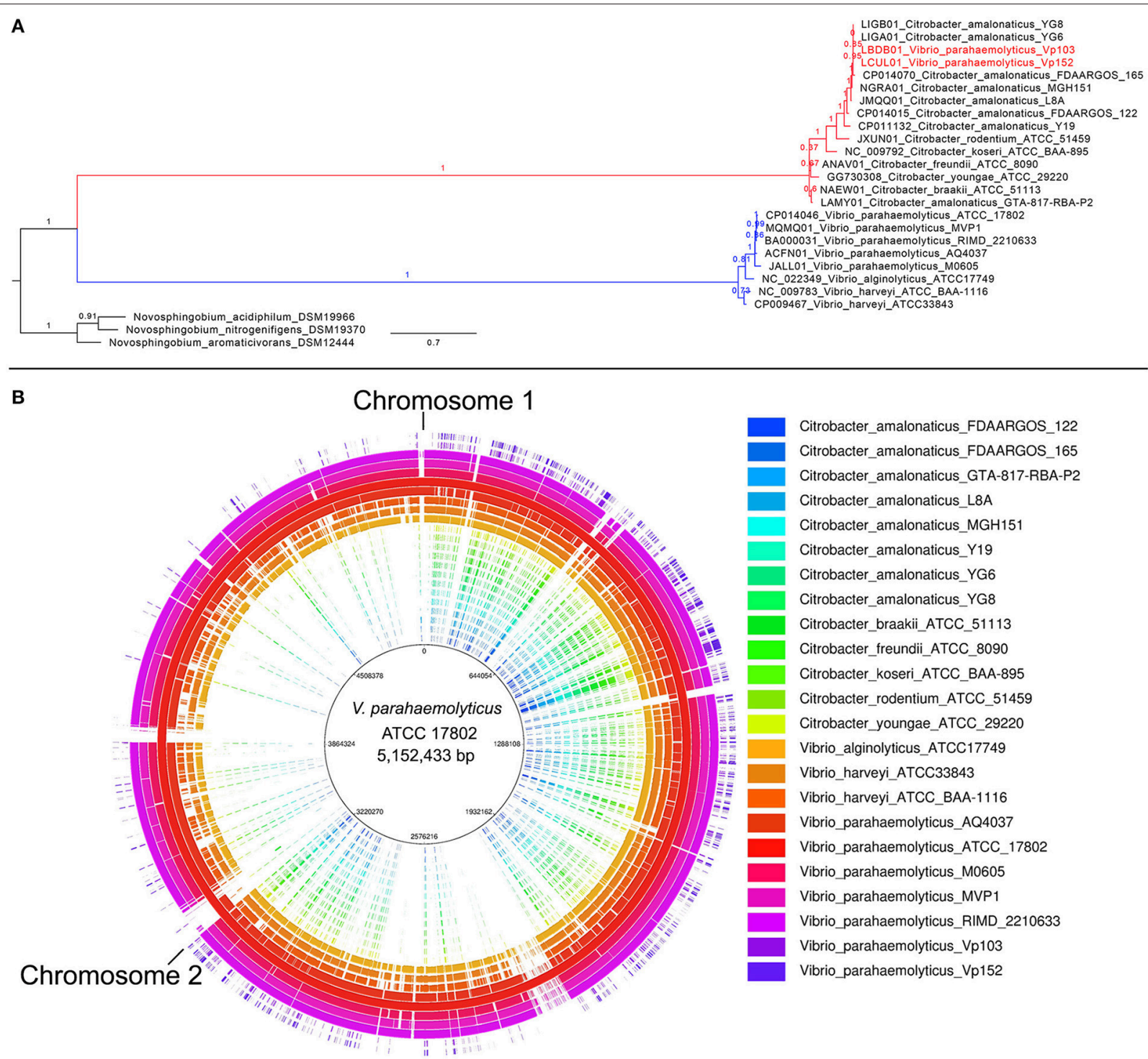

FIGURE 1 | Potential misidentification of strain VP152 to the genus Vibrio as revealed by phylogenomic analysis and whole genome nucleotide comparison with V. parahaemolyticus ATCC $17802^{\top}$. (A) Maximum likelihood tree constructed from the concatenated alignment of 400 universal proteins. The tree was rooted with members of the genus Novosphingobium as the outgroup. Node values indicate SH-like local branch support as implemented in FastTree2 and scale bar indicates the number of amino acid changes per site. (B) Whole genome comparisons of various Vibrio and Citrobacter strains against the complete genome of V. parahaemolyticus ATCC $17802^{\top}$. Regions exhibiting significant homology (BLASTN with an E-value cut-off of $1 \mathrm{E}^{-7}$ ) were colored based on strain label.

clustering of strains VP103 and VP152 with the two Citrobacter strains suggest potential sample mislabeling or barcode index misassignment during library preparation or sequencing.

Unfortunately, the authors did not describe any methodology associated with genome-based in-silico bacterial species validation in the data report to allow us to reproduce the identification of strain VP152 to the species V.parahaemolyticus. Given that the genome analysis of $V$. parahaemolyticus strain VP152 was based on the genome of a distantly related genus e.g. Citrobacter, it is unlikely that the biology interpretation in addition to the genome sequence reported in this study will be useful to the genomic study of $V$. parahaemolyticus or more generally the genus Vibrio.

\section{AUTHOR CONTRIBUTIONS}

HG, TA, TC, and CY performed data analysis. HG wrote the manuscript. All authors proofread the manuscript.

\section{FUNDING}

This research was supported by the Malaysian Ministry of Education (grant code FRGS/1/2016/STG05/MUSM/03/1) and by the Monash University Malaysia Tropical and Medicine Biology Multidisciplinary Platform (grant code 5140754-313). 


\section{REFERENCES}

Alikhan, N.-F., Petty, N. K., Ben Zakour, N. L., and Beatson, S. A. (2011). BLAST Ring Image Generator (BRIG): simple prokaryote genome comparisons. BMC Genomics 12:402. doi: 10.1186/1471-2164-12-402

Foo, S. M., Eng, W. W. H., Lee, Y. P., Gui, K., and Gan, H. M. (2017). New sequence types of Vibrio parahaemolyticus isolated from a Malaysian aquaculture pond, as revealed by whole-genome sequencing. Genome Announc. 5:e00302-17. doi: 10.1128/genomeA.00302-17

Kondo, H., Tinwongger, S., Proespraiwong, P., Mavichak, R., Unajak, S., Nozaki, R., et al. (2014). Draft genome sequences of six strains of Vibrio parahaemolyticus isolated from early mortality syndrome/acute hepatopancreatic necrosis disease shrimp in Thailand. Genome Announc. 2:e00221-14. doi: 10.1128/genomeA.00221-14

Letchumanan, V., Ser, H.-L., Chan, K.-G., Goh, B.-H., and Lee, L.-H. (2016a). Genome sequence of Vibrio parahaemolyticus VP103 strain isolated from shrimp in Malaysia. Front. Microbiol. 7:1496. doi: 10.3389/fmicb.2016.01496

Letchumanan, V., Ser, H.-L., Tan, W.-S., Ab Mutalib, N.-S., Goh, B.-H., Chan, K.-G., et al. (2016b). Genome sequence of Vibrio parahaemolyticus VP152 strain isolated from Penaeus indicus in Malaysia. Front. Microbiol. 7:1410. doi: $10.3389 /$ fmicb. 2016.01410

Segata, N., Börnigen, D., Morgan, X. C., and Huttenhower, C. (2013). PhyloPhlAn is a new method for improved phylogenetic and taxonomic placement of microbes. Nat. Commun. 4:2304. doi: 10.1038/ncomms3304
Soto-Rodriguez, S. A., Gomez-Gil, B., Lozano-Olvera, R., Betancourt-Lozano, M., and Morales-Covarrubias, M. S. (2015). Field and experimental evidence of Vibrio parahaemolyticus as the causative agent of acute hepatopancreatic necrosis disease of cultured shrimp (Litopenaeus vannamei) in Northwestern Mexico. Appl. Environ. Microbiol. 81, 1689-1699. doi: 10.1128/AEM. 03610-14

Yang, Y.-T., Chen, I. T., Lee, C.-T., Chen, C.-Y., Lin, S.-S., Hor, L.-I., et al. (2014). Draft Genome Sequences of four strains of Vibrio parahaemolyticus, three of which cause early mortality syndrome/acute hepatopancreatic necrosis disease in shrimp in China and Thailand. Genome Announc. 2:e00816-14. doi: 10.1128/genomeA. 00816-14

Conflict of Interest Statement: The authors declare that the research was conducted in the absence of any commercial or financial relationships that could be construed as a potential conflict of interest.

Copyright (c) 2018 Allnutt, Yan, Crowley and Gan. This is an open-access article distributed under the terms of the Creative Commons Attribution License (CC $B Y)$. The use, distribution or reproduction in other forums is permitted, provided the original author(s) and the copyright owner are credited and that the original publication in this journal is cited, in accordance with accepted academic practice. No use, distribution or reproduction is permitted which does not comply with these terms. 